\title{
ESTUDO DOS PARÂMETROS DE PROCESSO E EVOLUÇÃO MICROESTRUTURAL NA LAMINAÇÃO DE TIRAS A QUENTE VIA SIMULAÇÃO COMPUTACIONAL*
}

Antonio Lourenço Batista de Souza ${ }^{1}$ Rodrigo Bresciani Canto 2 Oscar Balancin ${ }^{3}$

\section{Resumo}

Neste trabalho, foi desenvolvido um modelo de elementos finitos para simular o primeiro passe da laminação de tiras a quente de aços C-Mn, durante a etapa de acabamento, via DEFORM ${ }^{T M} 3$. As dimensões e condições de processo foram obtidas a partir de dados industriais descritos na literatura. Utilizando-se o modelo termomecânico, foram feitas análises de sensibilidade por meio de alterações no fator de atrito e coeficiente de transferência de calor por condução, uma vez que não há um conhecimento preciso dos valores destes parâmetros para o processo simulado. Com isto, foram analisadas as alterações que a mudança destes parâmetros podem causar na carga e temperatura de laminação, perfil de deformação, fração recristalizada e tamanho de grão. O aumento do atrito provoca um aumento na carga de laminação, na homogeneidade do perfil de deformação e menor tamanho de grão. O aumento no coeficiente de transferência de calor, diminui a temperatura superficial da tira, o tamanho de grão e gera um aumento na carga de laminação. Assim, neste estudo foi mostrado a eficácia do modelo em predizer o comportamento do aço no processo de laminação a quente.

Palavras-chave: Laminação de tiras a quente; Simulação numérica; DEFORM 3D; Evolução microestrutural.

\section{STUDY OF PROCESS PARAMETERS AND MICROSTRUCTURAL EVOLUTION IN HOT STRIP ROLLING BY COMPUTER SIMULATION}

\section{Abstract}

In this work, a finite element model to simulate the first pass of the hot strip rolling of C-Mn steels was conducted by applying the software DEFORM ${ }^{T M} 3 D$. The process conditions and dimensions were obtained from industrial data described in the literature. Using a thermomechanical model, sensitivity analyzes were performed by changes in friction coefficient and heat transfer coefficient, since there is not a precise knowledge of the values of these parameters for the simulated process. Thus, the changes that the change of these parameters can cause in the load, rolling temperature, strain profile, recrystallized fraction and grain size were analyzed. The friction increased causes an increase in rolling load, in homogeneity of strain profile and reduced the grain size. The heat transfer coefficient increased leads to a decrease in surface temperature of strip, in grain size and an increase in rolling load. Thus, this study demonstrated the efficacy of the model to predict the behavior of the steel in hot rolling process.

Keywords: Hot strip rolling; Numerical simulation; DEFORM 3D; Microstructure evolution.

Engenheiro de Materiais, Mestre em Ciências e Engenharia de Materiais, Doutorando em Ciências e Engenharia de Materiais, PPG-CEM, UFSCar, São Carlos, SP, Brasil.

2 Engenheiro Mecânico, Doutor em Engenharia Mecânica, Professor Adjunto, DEMa, UFSCar, São Carlos, SP, Brasil.

3 Físico, Doutor em Engenharia Metalúrgica e de Materiais, Professor Titular, DEMa/PPG-CEM, UFSCar, São Carlos, SP, Brasil. 


\section{INTRODUÇÃO}

Aperfeiçoar o processamento dos aços é um dos principais focos da indústria metalúrgica devido à necessidade de materiais mais resistentes e produtos com menor peso, além das especificações cada vez mais rigorosas impostas pelos compradores. O conhecimento e controle da cinética dos fenômenos que ocorrem durante o processamento mecânico a quente como a recristalização, a precipitação e o crescimento de grão são mandatórios para o refinamento do tamanho dos grãos, o qual é um parâmetro que determina as propriedades dos produtos semiacabados. $\mathrm{Na}$ laminação a quente há a presença de gradientes de temperatura, grau e velocidade de deformação, e de composição química ao longo da espessura do laminado, desconhecimento sobre as condições reais na interface cilindro-tira e problemas para se coletar dados precisos, dificultando o controle do processo. Sendo assim, é recomendável que se conheçam os fenômenos metalúrgicos que podem ocorrer quando se define um esquema de passes para a laminação a quente [1].

A simulação numérica consiste em se reproduzir virtualmente o processo. Para isto torna-se necessário: o conhecimento detalhado das operações realizadas, 0 comportamento plástico do material, o comportamento microestrutural do material, a evolução da geometria das ferramentas e as interações ferramenta/material, além de métodos de cálculos adequados [2]. Utilizando-se o modelo termomecânico, foram feitas análises de sensibilidade por meio de alterações no fator de atrito e coeficiente de transferência de calor por condução, uma vez que não há um conhecimento preciso dos valores destes parâmetros para o processo simulado.

Condições de atrito na interface ferramenta/material podem influenciar o fluxo de metal, a formação de defeitos de superfície e internos, a tensão agindo sobre os cilindros e os valores de carga. Nas condições em que nenhum lubrificante é utilizado na interface e somente a camada de óxido presente na tira ou nos cilindros pode agir como um "separador" de camadas, o atrito é alto, como nos processos de laminação a quente. Valores do fator de atrito na faixa de 0,7 a 1 são prescritos para simulações de conformação a quente, quando nenhum lubrificante é utilizado [3].

Chen et al. [4] obtiveram uma relação entre o coeficiente de transferência de calor na interação ferramenta/material e a pressão média do laminador. Baseado nesta relação, encontraram que o coeficiente de transferência de calor por condução nos sete passes de laminação, no processo deles, variava de 18 a $50 \mathrm{~kW} \cdot \mathrm{m}^{-2} \cdot{ }^{\circ} \mathrm{C}^{-1}$. Li e Sellars [5] reportaram coeficientes de transferência de calor por condução na faixa de 8 a $18 \mathrm{~kW} \cdot \mathrm{m}^{-2} \cdot{ }^{\circ} \mathrm{C}^{-1}$ para processos de laminação a diferentes temperaturas e reduções. Fletecher and Beynon [6] listaram os coeficientes de transferência de calor por condução usado em laminação de aços, por diversos pesquisadores, reportando uma faixa de 2 a $200 \mathrm{~kW} \cdot \mathrm{m}^{-2} \cdot{ }^{\circ} \mathrm{C}^{-1}$. Todavia, Dyja e Korczak [7] usaram um coeficiente de transferência de calor por condução constante, $20 \mathrm{~kW} \cdot \mathrm{m}^{-2} \cdot{ }^{\circ} \mathrm{C}^{-1}$, em todos os passes de laminação.

O objetivo deste estudo foi, portanto, modelar o processo de laminação de tiras a quente acoplado à evolução microestrutural de um aço C-Mn, possibilitando analisar como os parâmetros do processo tais como carga, temperatura, deformação e tamanho de grão austenítico evoluem durante a laminação, utilizando o software comercial DEFORM ${ }^{\mathrm{TM}} 3 \mathrm{D}$, desenvolvido para análises de processos de conformação. Gerando uma ferramenta de simulação que possibilitará melhor adequação de determinadas variáveis de processo e, com isso, possibilitará a previsão de propriedades mecânicas finais, devido à previsão da evolução microestrutural. 


\section{MATERIAIS E MÉTODOS}

O comportamento plástico do material pode ser descrito por curvas de escoamento plástico, determinadas por meio de simulação física, replicando as condições de deformação (temperatura e taxas de deformação) do processamento industrial. Também, modelando essas curvas pode-se representar o material por meio de sua equação constitutiva. Neste trabalho utilizou-se a equação constitutiva (Equação 1) para descrever o comportamento plástico do aço a ser simulado.

$$
\dot{\varepsilon}=A \operatorname{senh}\left(\alpha \sigma_{p}\right)^{n} \exp \left(\frac{Q}{R T}\right)^{-1}
$$

sendo $\dot{\varepsilon}$ a taxa de deformação, $\boldsymbol{\sigma}_{\boldsymbol{p}}$ a tensão de pico, $\boldsymbol{T}$ a temperatura, $\boldsymbol{A}=\mathbf{5}, \mathbf{5 . 1 0} \mathbf{1 0}^{\mathbf{1 5}}$, $\alpha=0,013 \mathrm{MPa}, \mathrm{n}=5$ e $Q=358000 \mathrm{~kJ} . \mathrm{mol}^{-1}$.

Para a análise da evolução microestrutural foi utilizado o modelo de Johnson-MehlAvrami-Kolmogorov e as equações expostas na Tabela 1, após desenvolvimento matemático, foram utilizadas para obterem-se os parâmetros necessários de acordo com as equações do modelo apresentadas pelo programa.

Tabela 1. Equações que descrevem a evolução microestrutural dos grãos austeníticos $[8,9]$

\begin{tabular}{|c|c|}
\hline \multicolumn{2}{|c|}{ Equações } \\
\hline Deformação crítica & $\varepsilon_{c}=4,76 \cdot 10^{-4} \exp \left(\frac{8000}{T}\right)$ \\
\hline DRX & $\begin{array}{c}X=1-\exp \left[-0,693\left(\frac{\varepsilon-\varepsilon_{c}}{\varepsilon_{0,5}}\right)^{2}\right] \\
\varepsilon_{0,5}=1,144 \cdot 10^{-5} d_{0}^{0,28} \dot{\varepsilon}^{0,05} \exp \left(\frac{6420}{T}\right) \\
d_{D R X}=1,6 \cdot 10^{4} \cdot Z^{-0,23}\end{array}$ \\
\hline MDRX & $\begin{array}{c}X=1-\exp \left[-0,693\left(\frac{t}{t_{0,5}}\right)^{1,5}\right] \\
t_{0,5}=1,1 \cdot Z^{-0,8} \exp \left(\frac{240000}{R T}\right) \\
d_{M D R X}=2,6.10^{4} \cdot Z^{-0,23}\end{array}$ \\
\hline SRX & $\begin{array}{c}X=1-\exp \left[-0,693\left(\frac{t}{t_{0,5}}\right)^{1}\right] \\
t_{0,5}=2,3 \cdot 10^{-15} \varepsilon^{-2,5} d_{0}^{2} \exp \left(\frac{230000}{R T}\right) \\
d_{S R X}=343 \varepsilon^{-0,5} d_{0}^{0,4} \exp \left(\frac{-45000}{R T}\right)\end{array}$ \\
\hline Crescimento de grão & $d^{7}=d_{0}^{7}+1,45.10^{27} t \cdot \exp \left(\frac{-400000}{R T}\right)$ \\
\hline
\end{tabular}

A primeira etapa do trabalho envolve a caracterização do processo industrial a ser estudado. Nesta etapa do trabalho foram utilizados os parâmetros medidos em uma planta industrial e descritos na literatura [10]. As condições de processamento estão apresentadas na Tabela 2. 
Tabela 2. Dados de entrada do processo industrial para o primeiro passe [10]

\begin{tabular}{|c|c|c|c|c|c|c|}
\hline $\begin{array}{c}\text { Espessura } \\
\text { de entrada }\end{array}$ & $\begin{array}{c}\text { Espessura } \\
\text { de saída }\end{array}$ & $\begin{array}{c}\text { Largura } \\
\text { da tira }\end{array}$ & $\begin{array}{c}\text { Temperatura } \\
\text { da tira }\end{array}$ & $\begin{array}{c}\text { Raio do } \\
\text { cilindro }\end{array}$ & $\begin{array}{c}\text { Velocidade } \\
\text { do cilindro }\end{array}$ & Carga \\
\hline $30,60 \mathrm{~mm}$ & $17,33 \mathrm{~mm}$ & $1264 \mathrm{~mm}$ & $987^{\circ} \mathrm{C}$ & $394 \mathrm{~mm}$ & $33,90 \mathrm{rpm}$ & $21,57 \mathrm{MN}$ \\
\hline
\end{tabular}

Foi desenvolvido um modelo do método dos elementos finitos de análise termomecânica acoplada com a microestrutura, com o auxílio das leis de evolução microestrutural e dos modelos matemáticos de deformação a quente. $\mathrm{O}$ modelo possui uma boa concordância com o processo industrial descrito [11]. Para isso,

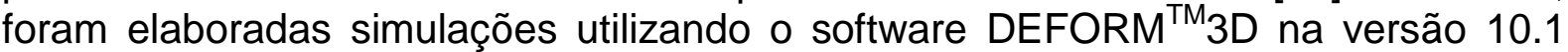
variando os parâmetros e as condições de processo de acordo com a Tabela 3.

Tabela 3. Dados de entrada variando as condições de processo

\begin{tabular}{|c|c|c|c|}
\hline Simulação & $\begin{array}{c}\text { Fator de } \\
\text { atrito }\end{array}$ & $\begin{array}{c}\text { Coeficiente de transferência de } \\
\text { calor por condução }\left(\mathbf{k W} \cdot \mathbf{m}^{-2}{ }^{\circ} \mathbf{C}\right)\end{array}$ & $\begin{array}{c}\text { Tamanho de } \\
\text { grão inicial }(\boldsymbol{\mu m})\end{array}$ \\
\hline S1 & 0,5 & 40 & 100 \\
\hline S2 & 0,7 & 40 & 100 \\
\hline S3 & 1,0 & 40 & 100 \\
\hline S4 & 0,5 & 20 & 100 \\
\hline S5 & 0,5 & 80 & 100 \\
\hline
\end{tabular}

Neste trabalho o cilindro foi definido com comportamento rígido e a tira com comportamento plástico, sendo assim, a deformação elástica do cilindro foi levada em consideração por meio da análise desenvolvida por Hitchcock para 0 achatamento dos cilindros, tendo este valor empregado nas simulações ( $R^{\prime}=405$ $\mathrm{mm}$ ).

Devido à simetria do processo de laminação de tiras a quente, a simulação pode ser conduzida pela modelagem de um quarto da tira (metade da largura e metade da espessura), como esquematizado na Figura 1 . O número de elementos inicial na tira foi de 7200 elementos. Um tamanho de grão austenítico inicial de $100 \mu \mathrm{m}$ foi assumido para todos os elementos. Os planos de simetria são definidos de acordo com a geometria dos objetos e as áreas de transferência de calor correspondem às faces que estão expostas ao ambiente e aos contatos com os ferramentais.

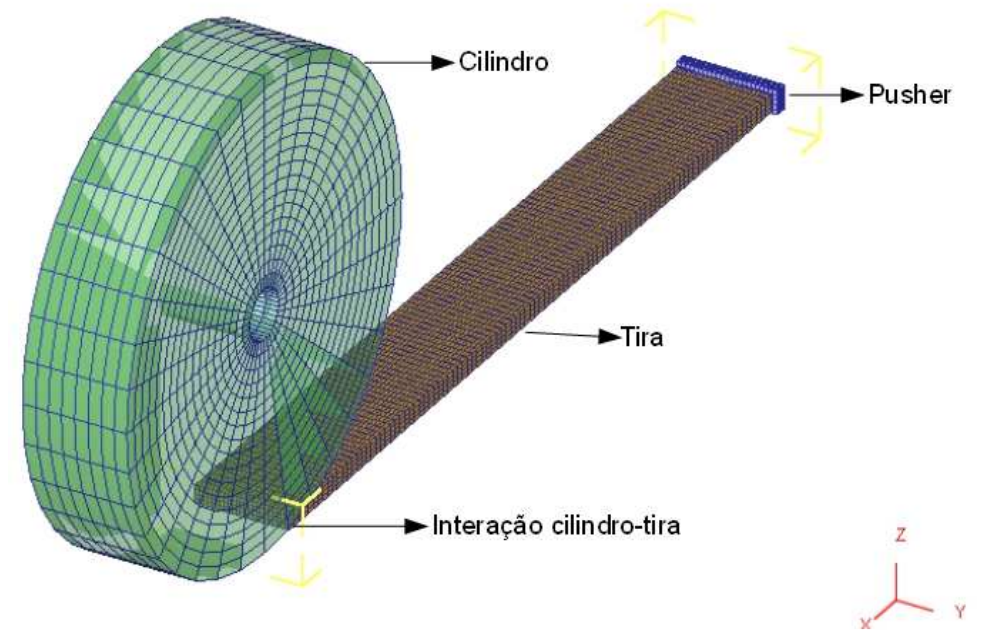

Figura 1. Representação esquemática do modelo geométrico.

Os controles de simulação consistem nas informações gerais que o programa utiliza para identificar o problema. Inclui instruções sobre os métodos que o sistema deve 
usar para resolver o problema, incluindo as condições do ambiente de processamento, que processos físicos devem ser modelados, quantos passos devem ser usados para modelar o processo, entre outros. Todos estes dados foram selecionados de acordo com o que já está pré-definido pelo software para o processo de laminação e estão listados na Tabela 4.

Tabela 4. Dados de entrada para o controle de simulação

\begin{tabular}{|l|l|}
\hline Principal & SI \\
\hline $\begin{array}{l}\text { Sistema de Unidades } \\
\text { Tipo de Simulação } \\
\text { Modos de Simulação }\end{array}$ & $\begin{array}{l}\text { Lagrangeano Incremental } \\
\text { Deformação, Transferência de Calor (Grão) }\end{array}$ \\
\hline Controle de Passos \\
\hline $\begin{array}{l}\text { Número de passos da simulação } \\
\text { Incremento para salvar passos }\end{array}$ & 5000 \\
Incremento de tempo & 10 \\
\hline Iteração & $0,0005 \mathrm{~s}$ \\
\hline Solucionador Deformação & Esparso \\
Método de Iteração & Iteração Direta \\
Solucionador Temperatura & Esparso \\
Erro Limite Velocidade & 0,01 \\
Erro Limite Força & 0,05 \\
\hline Condições de Processo & $30^{\circ} \mathrm{C}$ \\
\hline Temperatura Ambiente & $0,02 \mathrm{~kW} \cdot \mathrm{m}^{-2} \cdot{ }^{\circ} \mathrm{C}^{-1}$ \\
Coeficiente de Convecção
\end{tabular}

No controle de passos definem-se o número total de passos da simulação, o incremento para salvar passos e como vai ser o passo da simulação. $O$ incremento para salvar passos indica de quantos em quantos passos devem ser gravados os resultados da simulação no arquivo de base de dados. O passo da simulação pode ser definido por incremento de tempo ou por incremento de deslocamento, que associado com o número total de passos da simulação determina a duração do processo simulado. Adicionalmente, o critério de remalhamento automático da tira foi adotado, em vista da possibilidade de surgir malhas severamente distorcidas, causada pela extensa deformação durante a laminação a quente.

\section{RESULTADOS E DISCUSSÃO}

\subsection{Fator de Atrito}

O fator de atrito foi variado de 0,5 a 1,0 (simulações S1, S2 e S3) e o efeito sobre a carga e temperatura de laminação foi estudado. Um aumento no fator de atrito de 0,5 para 1,0 causa um aumento na carga de laminação em torno de $20 \%$, como pode ser observado na Figura 2a. De acordo com a Figura 2b, observa-se que o valor da carga de laminação simulado que mais se aproxima do valor medido na planta industrial é o da simulação que utiliza fator de atrito igual a 0,5 (simulação S1), que pode estar relacionado com a possível transição do atrito de agarramento para o de deslizamento provocada pela carepa superficial, no caso da primeira cadeira. O efeito do fator de atrito na temperatura de laminação foi desprezível.

Observou-se que a variação do fator de atrito também gera uma variação no perfil da deformação efetiva. A deformação efetiva, na direção da espessura da tira, tornase menos homogênea (Figura 3). A deformação no centro da tira não sofre 
variações significativas, ao contrário do que ocorre na superfície, que experimenta as deformações máximas (Figura 4).

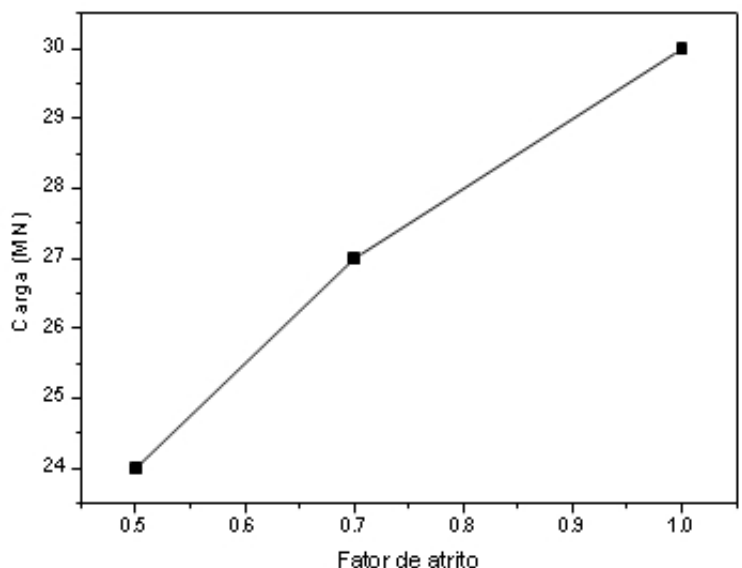

(a)

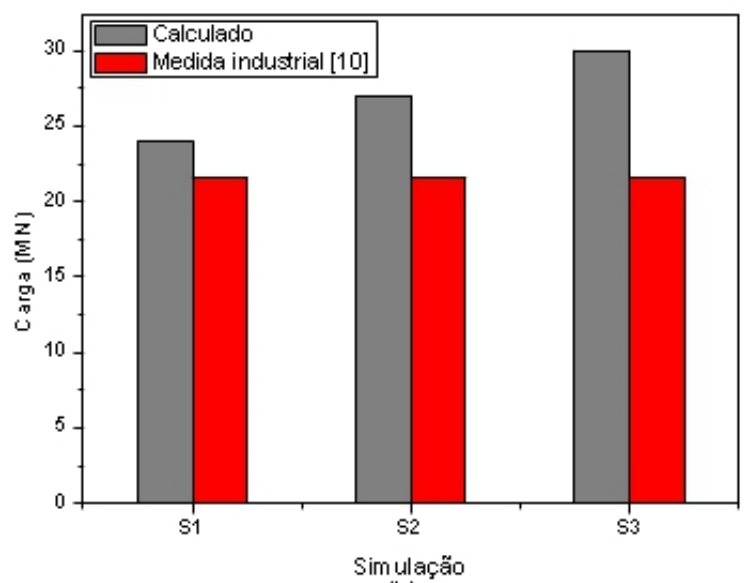

(b)

Figura 2. Gráfico da carga de laminação (a) em função do fator de atrito e (b) comparação entre as cargas de laminação calculadas pelo modelo e a carga medida industrialmente [10].

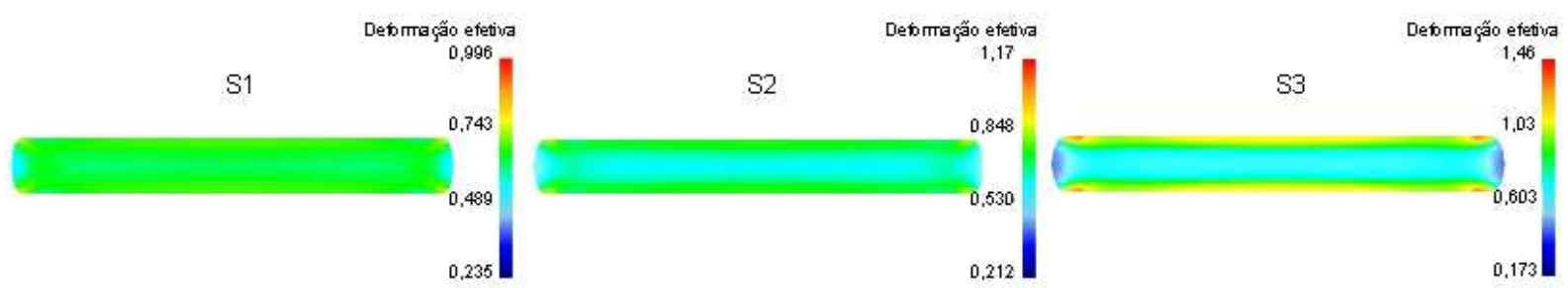

Figura 3. Perfil de deformação na seção transversal da tira nas simulações $S 1$, S2 e S3 em função do atrito.
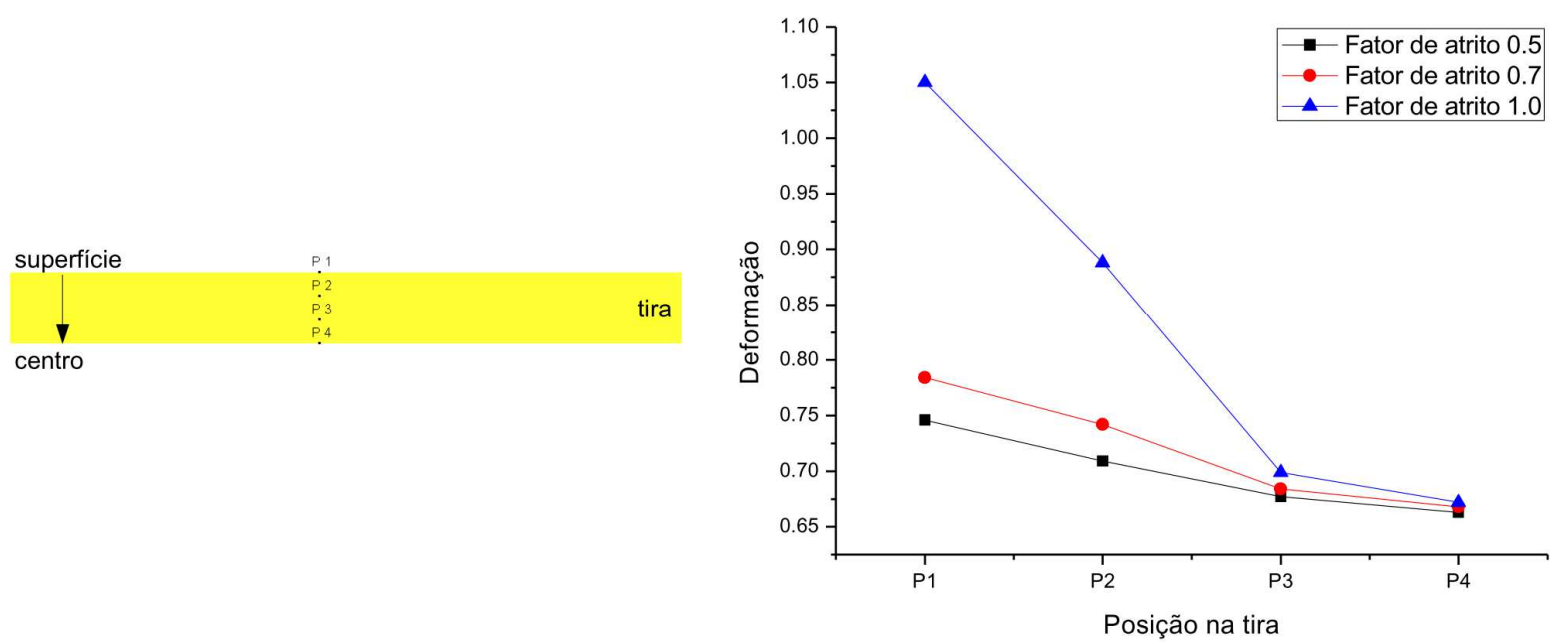

Figura 4. Um quarto da seção transversal da tira com as posições de coleta dos valores calculados e gráfico da deformação, para diversos pontos ao longo da espessura da tira, em função do atrito.

Uma vez que o perfil de deformações é afetado pelo fator de atrito, a fração recristalizada também sofre alterações. Como pode ser visto na Figura 5 com maior atrito, a fração recristalizada, principalmente na superfície da tira é mais efetiva, devida às maiores deformações nessa região.

A evolução do tamanho de grão também sofre alterações. Na Figura 6 observa-se que o tamanho de grão austenítico tende a diminuir com a presença de maior atrito 
na interface cilindro-tira, principalmente na região da superfície da tira, que cai de 26 $\mu \mathrm{m}$ para $20 \mu \mathrm{m}$, o que está relacionado com a influência do atrito na deformação aplicada e com a taxa de deformação nesta região. Já na região do centro da tira, o tamanho de grão austenítico passa de $30 \mu \mathrm{m}$ para $27 \mu \mathrm{m}$ com o aumento do atrito.

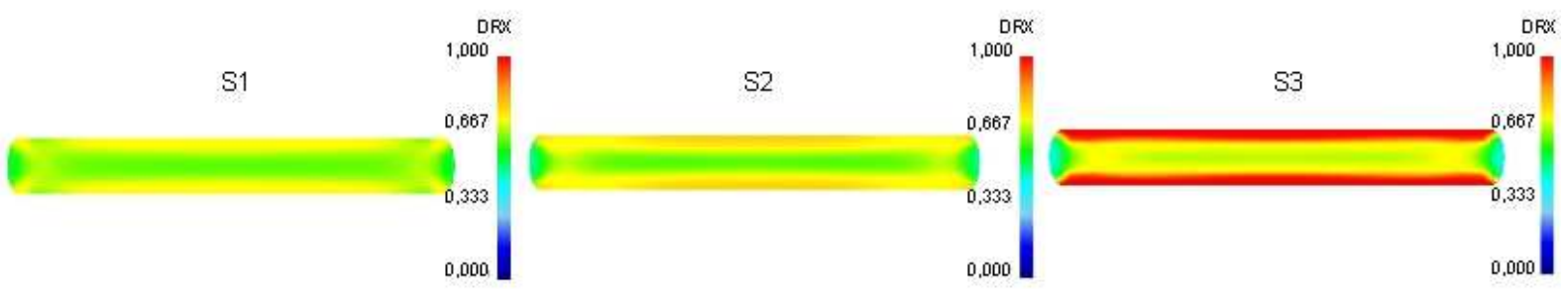

Figura 5. Perfil de recristalização dinâmica na seção transversal da tira nas simulações S1, S2 e S3 em função do atrito.

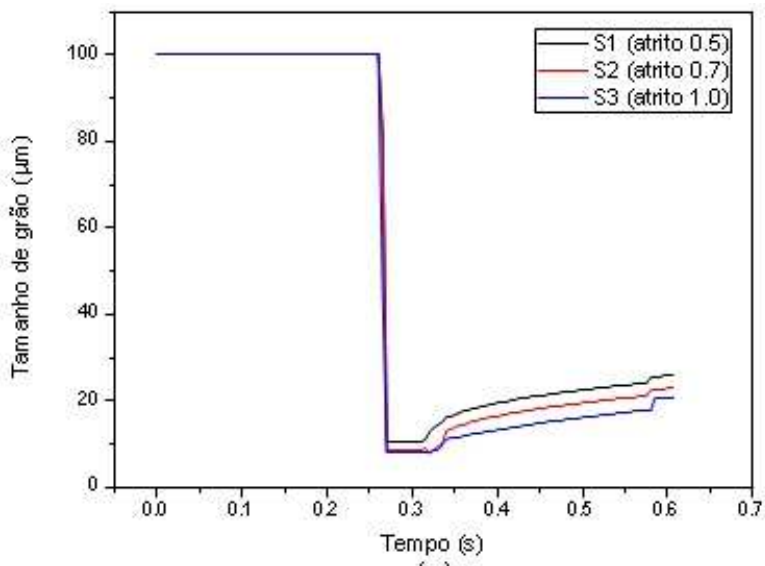

(a)

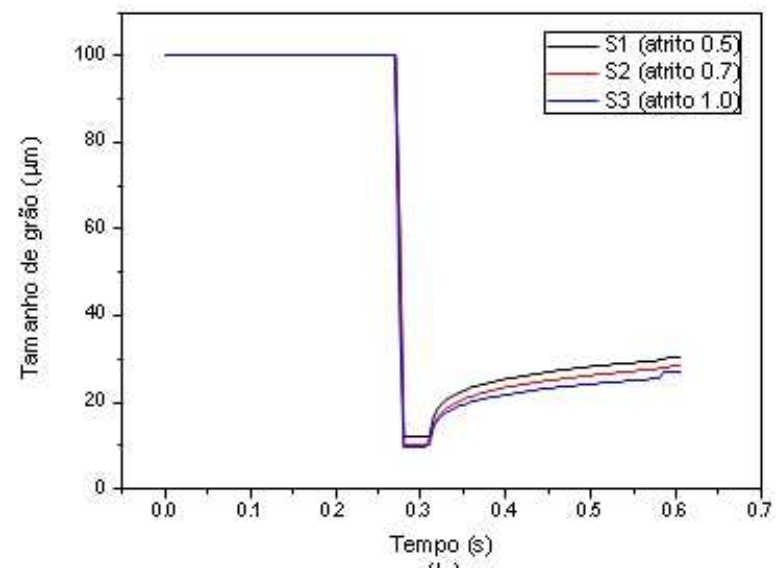

(b)

Figura 6. Gráfico da evolução do tamanho de grão austenítico em função do fator de atrito para (a) regiões na superfície da tira e $(b)$ regiões no centro da tira.

\subsection{Coeficiente de Transferência de Calor por Condução}

Variou-se o coeficiente de transferência de calor de 20 a $80 \mathrm{~kW} \cdot \mathrm{m}^{-2} \cdot{ }^{\circ} \mathrm{C}^{-1}$ (simulações S4, S1 e S5) e analisou-se o efeito causado na temperatura e carga de laminação. Como resultado, obteve-se um decréscimo em torno de $5 \%$ na temperatura de laminação com o aumento do coeficiente de transferência de calor de 20 para 80 $\mathrm{kW} \cdot \mathrm{m}^{-2} \cdot{ }^{\circ} \mathrm{C}^{-1}$ durante a aplicação da deformação, o valor da temperatura calculada que mais se aproxima com o valor medido na planta industrial é o da simulação S1 $\left(40 \mathrm{~kW} \cdot \mathrm{m}^{-2} .{ }^{\circ} \mathrm{C}^{-1}\right)$ como é mostrado na Figura 7.

A carga de laminação também sofre alterações com o aumento do coeficiente de transferência de calor por condução, uma vez que menores temperaturas de laminação ocasionam maiores cargas, com isso, foi observado um aumento na carga em torno de 10\% quando comparadas as simulações S4 e S5. Na Figura 8 é apresentada a comparação dos valores de carga calculado para cada coeficiente de transferência de calor com a carga medida na planta industrial.

Não houve mudanças significativas no perfil de deformação, o mesmo foi observado para a fração recristalizada, como era esperado. No entanto, o tamanho de grão diminui com o aumento do coeficiente de transferência de calor, visto que as trocas de calor são mais efetivas e há uma queda na temperatura da tira, principalmente nas regiões da superfície, levando a um menor crescimento de grão, que pode ser observado na Figura 9. 


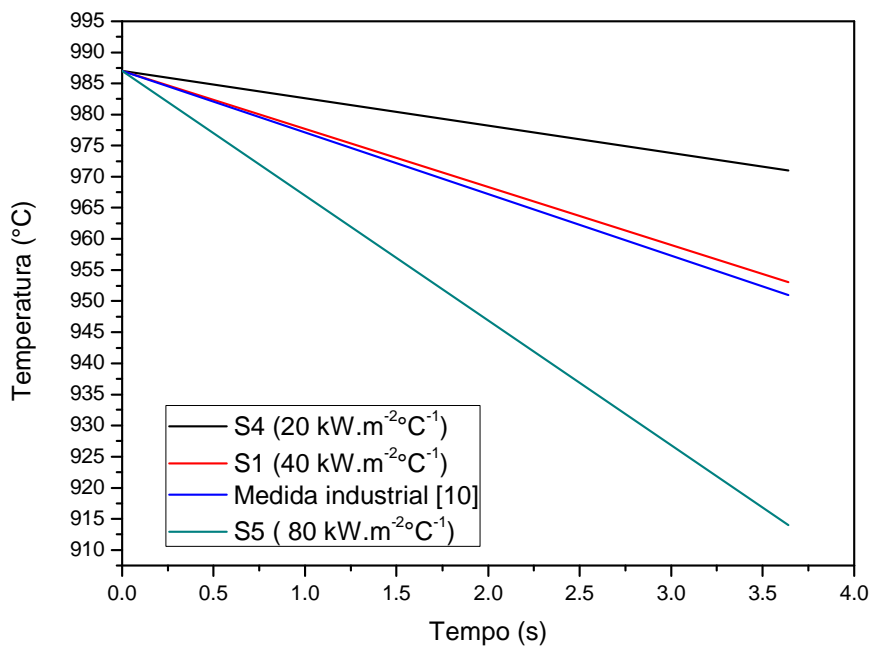

Figura 7. Comparação da evolução da temperatura superficial da tira entre as simulações $\mathrm{S} 1$, S4 e S5 e a medida na planta industrial [10].

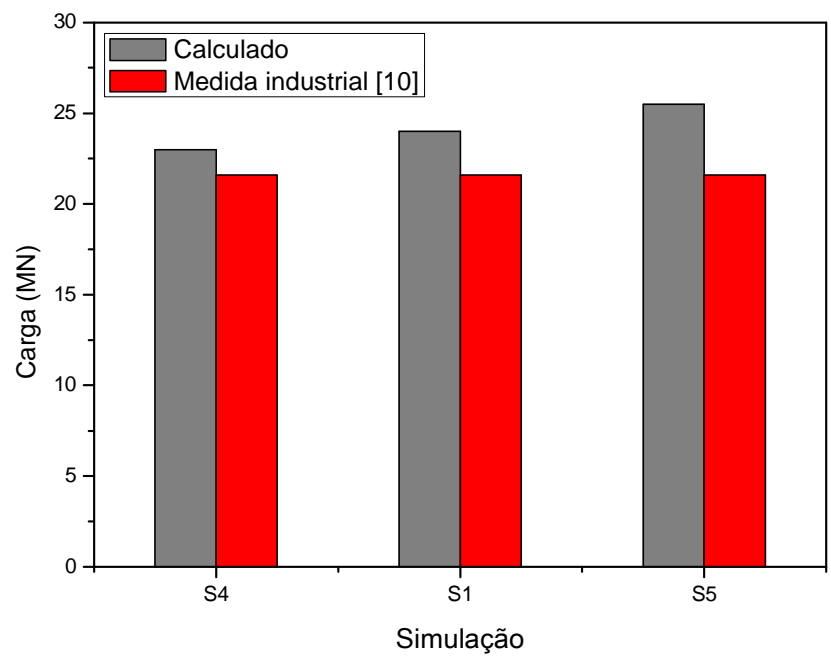

Figura 8. Comparação entre as cargas de laminação calculadas pelo modelo e a carga medida industrialmente [10] em função do coeficiente de transferência de calor.

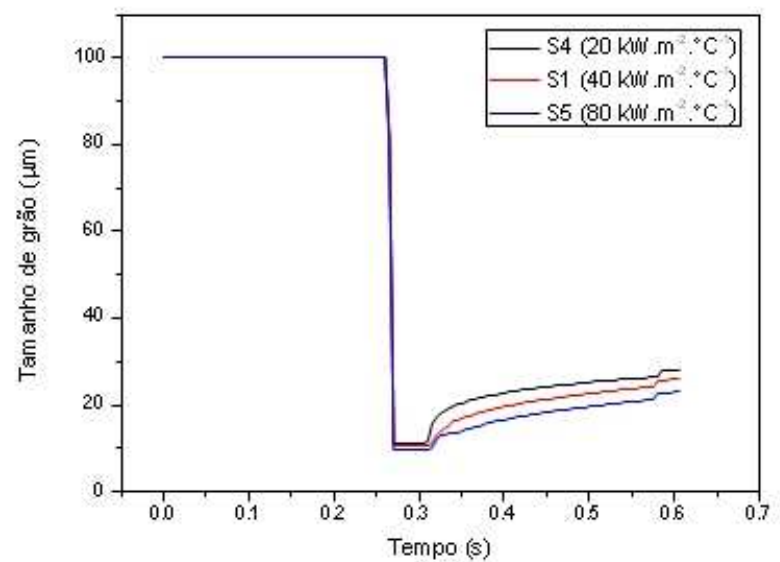

(a)

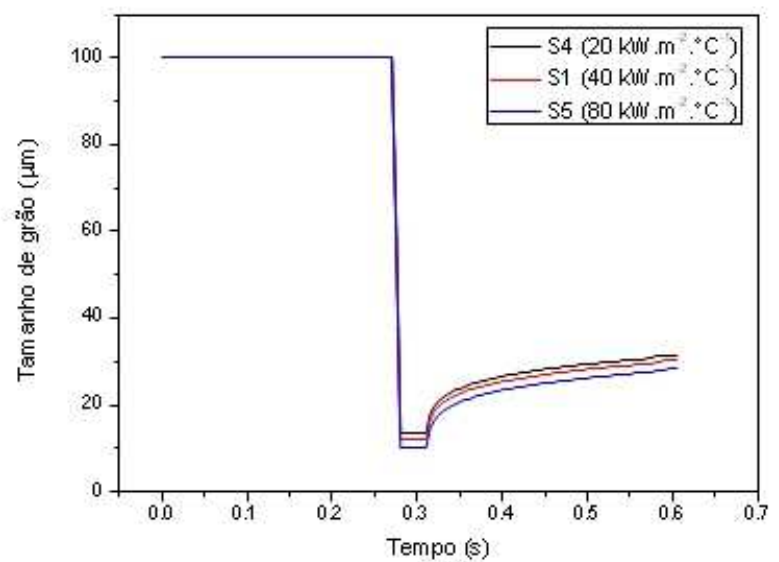

(b)

Figura 9. Gráfico da evolução do tamanho de grão austenítico em função do coeficiente de transferência de calor para (a) regiões na superfície da tira e (b) regiões no centro da tira.

De acordo com o gráfico da Figura 9, o tamanho de grão cai de $28 \mu \mathrm{m}$ para $23 \mu \mathrm{m}$, na região da superfície da tira, com o aumento do coeficiente de transferência de 
calor, devido a maiores quedas de temperatura, influenciando no crescimento de grão. Na região do centro da tira o tamanho de grão cai de $31 \mu \mathrm{m}$ para $28 \mu \mathrm{m}$.

Com base nessas análises, fica evidente que há um gradiente no tamanho de grão ao longo da espessura da tira, devido as influências dos gradientes de temperatura, deformação e taxa de deformação, e que são possíveis de se calcular através do modelo proposto.

\section{CONCLUSÃO}

O estudo da influência do fator de atrito e do coeficiente de transferência de calor por condução mostrou que a precisão e fidelidade dos dados industriais coletados são fundamentais para a simulação correta e análise da evolução microestrutural que ocorre na tira.

O fator de atrito exerce influência sobre a carga de laminação e principalmente sobre o perfil de deformação, causando modificação na fração recristalizada e tamanho de grão austenítico.

O coeficiente de transferência de calor por condução na interface cilindro-tira atua na carga e temperatura de laminação, influenciando também o crescimento de grão, no entanto, um aumento de quatro vezes do coeficiente não causa uma modificação brusca nas variáveis analisadas, visto que o tempo de contato entre a tira e o cilindro é curto.

Fica evidente a presença de gradiente de deformação, influenciando os mecanismos de recristalização e crescimento de grão ao longo da espessura da tira.

Pequenas variações nos parâmetros de processo, como a temperatura de laminação, podem alterar de forma significativa toda a evolução microestrutural que acontece durante o processo, além de refletir na evolução das cargas de laminação.

\section{Agradecimentos}

Os autores agradecem ao suporte financeiro dado pela Coordenação de Aperfeiçoamento de Pessoal de Nível Superior - Capes.

\section{REFERÊNCIAS}

1 Gorni AA, Silva MRS. Modelamento da evolução microestrutural durante a laminação de tiras a quente de aços microligados ao nióbio. In: 49 Seminário de Laminação Processos e Produtos Laminados e Revestidos, 2012, p. 703-712.

2 Souza ALB, Canto RB, Balancin O. Laminação a quente: Simulação numérica acoplada com a evolução microestrutural. Revista ABM - Metalurgia, Materiais \& Mineração. 2014; 70; 499-503.

3 Kobayashi S, Oh SI, Altan T. Metal forming and the finite-element method. New York: Oxford University Press; 1989.

4 Chen W, Samarasekera I, Hawbolt E. Fundamental phenomena governing heat transfer during rolling. Metallurgical Transactions A. 1993; 24(6): 1307-1320.

$5 \mathrm{Li} \mathrm{Y,} \mathrm{Sellars} \mathrm{C,} \mathrm{Comparative} \mathrm{investigations} \mathrm{of} \mathrm{interfacial} \mathrm{heat} \mathrm{transfer} \mathrm{behaviour} \mathrm{during}$ hot forging and rolling of steel with oxide scale formation. Journal of Materials Processing Technology. 1998; 80: 282-286.

6 Fletcher J, Beynon J. Heat transfer conditions in roll gap in hot strip rolling. Ironmaking \& steelmaking. 1996; 23(1): 52-57. 
7 Dyja $\mathrm{H}$, Korczak $\mathrm{P}$. The termal-mechanical and microstructural model for the FEM simulation of hot plate rolling. Journal of Materials Processing Technology. 1999; 92: 463-467.

8 Hodgson P, Gibbs R. A mathematical model to predict the mechanical properties of hot rolled C-Mn and microalloyed steels. ISIJ international. 1992; 32(12): 1329-1338.

9 Senuma T, Yada H. Microstructural evolution of plain carbon steels in multiple hot working. In Proceedings of the Riso International Symposium on Metallurgy and Materials Science, 7th; 1986; RISO National Laboratory, Roskilde, Dinamarca. p. 547552.

10 Siciliano Jr F. Mathematical Modeling of the Hot Strip Rolling of Nb Microalloyed Steels [PhD Thesis]. Montreal, Canada: McGill University; 1999.

11 Souza ALB, Canto RB, Balancin O. Análise da evolução microestrutural na laminação de tiras a quente usando método de elementos finitos. In: $51^{\circ}$ Seminário de Laminação - Processos e Produtos Laminados e Revestidos. 2014 [acesso em 13 março 2015]. Disponível em http://www.abmbrasil.com.br/anais/laminacao-2014. 